\title{
The flexible body modeling and simulation study of the planet gear based on RecurDyn
}

\author{
Yujie $\mathrm{Hou}^{1, \mathrm{a}}$, Guangbin Feng ${ }^{2}$, Huagang Sun ${ }^{2}$ and Chiyu $\mathrm{HaO}^{2}$ \\ ${ }^{1}$ Ordnance Engineering College,Shijiazhuang 050003,China \\ ${ }^{2}$ Ordnance Technical Institution,Shijiazhuang 050003,China \\ a1843480237@qq.com
}

Keywords:RecurDyn ,planet gear ,flexible.

\begin{abstract}
RecurDyn,in the planet gear of two freedom degree as an example ,establishing the flexible dynamic model,then,the dynamic simulation analysis.Draw out the stress distribution,the simulation and stress distribute results are closed to theoretical calculation values.The study results show that a reliable reference for planet gear processing manufacturing ,material stress analysis ,maintenance and life prediction was provide.
\end{abstract}

\section{Introduction}

Gear transmission is one of the most common transmission mode in engineering .With the rapid development of the gear processing and manufacturing technology. Planetary gear transmission technology more and more high performance requirements. For instance, improving the bearing capacity of the gear,transmission efficiency,losing gear weight and increasing the ratio. Planet gear is wildly used in a certain type planet gear transmission box. It plays a key role in the power transmission and the direction of change.

In the actual situation, planet gear is prone to have lots of problems, for instance ,wear and tear,tooth fracture,etc. In the processing and manufacturing process,the different technological level will cause the processing of gear strength different. So,in order to improve the gear strength and service life,it is necessary for the flexible analysis of the planet gear. This article mainly establish flexible model of the planet gear of a certain type of planet gear and analysis the stress distribution based RecurDyn, the study results can provide a reference for the design and manufacture of the planet gear.

\section{The theory of gear transmission}

\subsection{The structure of the planet gear of certain type transmission box}

As shown in figure 1, the structure of the planet gear of certain type transmission box is sun gear ,planet gear,planet carrier,gear ring and planet carrier.In order to facilitate analysis, use the center gear 1,center gear 2 replace the sun gear,gear ring.

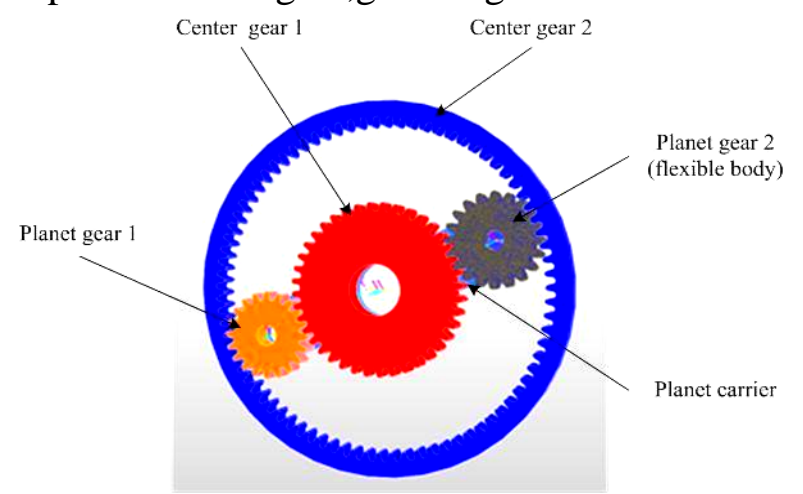

Fig. 1 The structure of the planet gear

\section{2 transmission theory}

The transfer between the gear by contact,contact force is very important in the gear transmission , 
Gear contact force $F_{N}[1]$ is follow:

$$
F_{N}=k \delta^{m_{1}}+c \frac{\dot{\delta}}{|\dot{\delta}|}|\dot{\delta}|^{m_{2}} \delta^{m_{3}}
$$

From the above formula: $m_{1}$ is stiffness coefficient; ${ }^{c}$ is damping coefficient; $\delta$ is contact depth; $\delta$ is relative velocity of first catch; $m_{1} 、 m_{2} 、 m_{3}$ represent the stiffness,the damping index,the index of the indentation (by default $m_{3}$ is 0 ).

\section{Establish the flexible model of dynamics simulation.}

\subsection{Flexible theory}

The flexible is variant[2], the elastic will be disperse the element,creating many nodes. In the process of deformation of flexible body,the position of each node is changing,so,relative coordinates are not suitable for the kinematic of flexible body. So ,it is necessary for introducing elastic coordinate(floating coordinate).Considering the location of the node changes before and after ,the direction and mode. Decare Coordinate: $X=(x, y, z)$;Euler angle: $\psi=(\psi, \theta, \varphi)$;the modal coordinates: $q=\left(q_{1}, q_{2} \ldots q_{n}\right)^{T}$. So,the generalized coordinate of the flexible is $\zeta=\{x, \psi, q\}^{T}=\left\{x, y, z, \psi, \theta, \varphi, q_{i}(i=1 \ldots n)\right\}^{T}$, the position vector of the any point about the flexible body is $r_{i}=X_{0}+A\left(s_{i}+\varphi_{i} q\right), X_{0}$ is the origin of floating coordinate system vector in the inertial coordinate system, A is the direction cosine matrix , $s_{i}$ is the vector of the complete flexible in the floating coordinate, $\varphi_{i}$ is the assuming modal matrix of the meeting the requirements of this base vector, $q$ is deformation of the generalized coordinates.

So, the velocity of the node is $V_{\mathrm{i}}=\dot{r}_{i}=\left[E-A\left(s_{i}+\varphi_{i} q\right) B+A \varphi_{\mathrm{i}}\right] \dot{\xi}$, B is transfer matrix between the angular velocity vector and time mathematical inverse of the Euler.Ultimately, motion differential equation of the flexible body is:

$$
M \ddot{\xi}+\dot{M} \dot{\xi}-\frac{1}{2}\left[\frac{\partial M}{\partial \xi} \dot{\xi}\right]+K \xi+f_{g}+D \dot{\xi}+\left[\frac{\partial \psi}{\partial \xi}\right]^{T} \lambda=Q
$$

From the above formula: $\dot{\xi}, \ddot{\xi}$ is the flexible body first order derivative of the coordinates about the time and the second order derivative , $M$ is flexible body derivative of the mass matrix about the time. The same true for $\frac{\partial M}{\partial \xi}$ and $\frac{\partial \psi}{\partial \xi}$.The formula describe the particle trajectories of the flexible body,greatly.As well ,it provide a theory for the model and simulation.

\subsection{Establish the flexible model}

Establishing the flexible model is necessary for gear transmission analysis,in the rigid contact ,we can not analysis the stiffness,deformation,stress and strain. Firstly, set up model of gear mesh according to drawing of a certain type planetary gear transmission box in PTC CREO. Save the model as "x_t”format. Secondly,importing model of saved into the RecurDyn .Thirdly,set the constraint,contact,etc.

The flexible model is follow: 


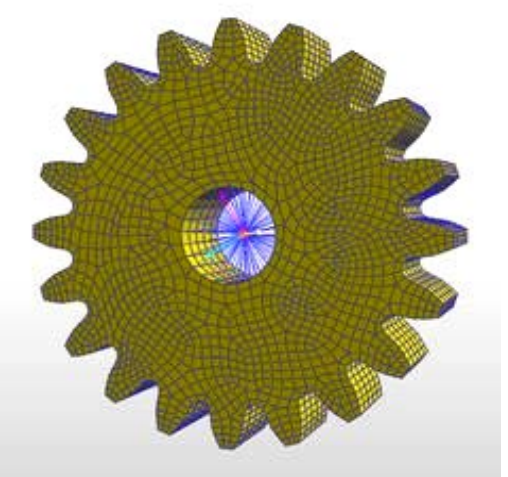

Fig.2 Flexible model of the planet gear

\section{Simulate and analysis}

Set two centers velocity are $360 \mathrm{r} / \mathrm{min}$ and $120 \mathrm{r} / \mathrm{min}$,teeth respectively $\mathrm{Z} 1=40, \mathrm{Z} 2=80$, so the planet carrier's velocity is 200rad/s according to the theoretical arithmetic.Choose Analysis $>>$ Simulation Type $>>$ Dynamic/Kinematic Analysis,set the end time 1.5s ,the simulation steps is 300,define drawing the number of sampling point data is 5 ,then simulate ,the results as follow:

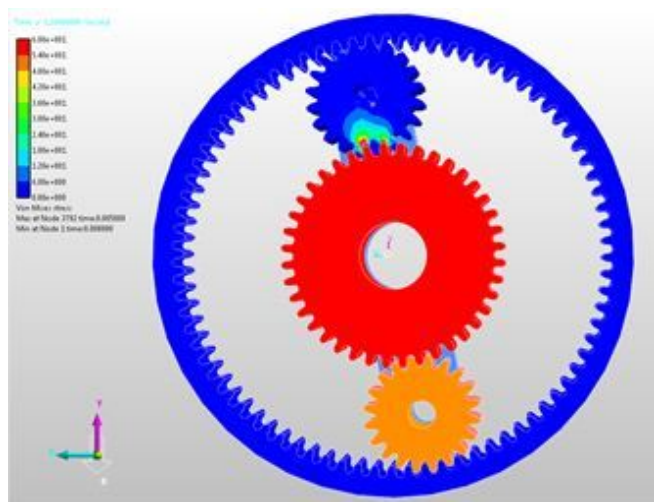

Fig.3 Von Mises nephogram of the planet gear

Analysis: From the above Von Mises nephogram of the planetary gear is know,the maximum of the stress is $2.89 \mathrm{e}+003 \mathrm{~Pa}$, the minimum of the stress is $1.16 \mathrm{e}+003 \mathrm{~Pa}$, the max of the stress is2890Pa.We can according to distribution of the stress on the Von Mises nephogram ,targeted for heat treatment of metals and surface,increase the mechanical properties of the gear materials.

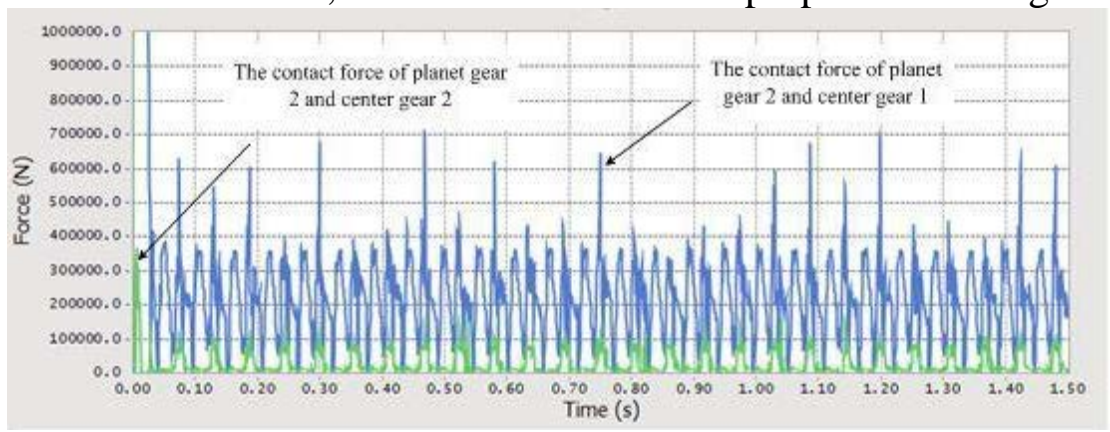

Fig.4 Flexible contact force

Analysis:To facilitate analysis ,assume F1 instead of the contact force of the planet gear 2 and center gear 2;F2 instead of the contact force planet gear 2 and center gear 1.From the above curve know, on the whole F1 is bigger than F2, F1 is relatively stable than F2,just at the start of the movement (the time is $0 \mathrm{~s}$ ), F1 is $1 \times 10^{6} \mathrm{~N}$,but F2 is none, this suggest that the flexible contact force is related to the gear velocity,meshing method,etc[3]. Because the gear's velocity is greater, the gear what meshing with it variant is greater. Aim at a certain type planet gear transmission box,the sun gear fault rate is more ,when disassemble the transmission box ,firstly, the sun gear be should considered check. 
One the other hand,consider the micro deformation and the actual working condition, the simulation results of the flexible body are closed to the actual working condition. We should forecast fault rate of the center gear at fixed period or collect the signal about the center gear, analysis the signal according the curve of the flexible contact force,in order to predict the fault and take a effective measure to solve the problems in time.

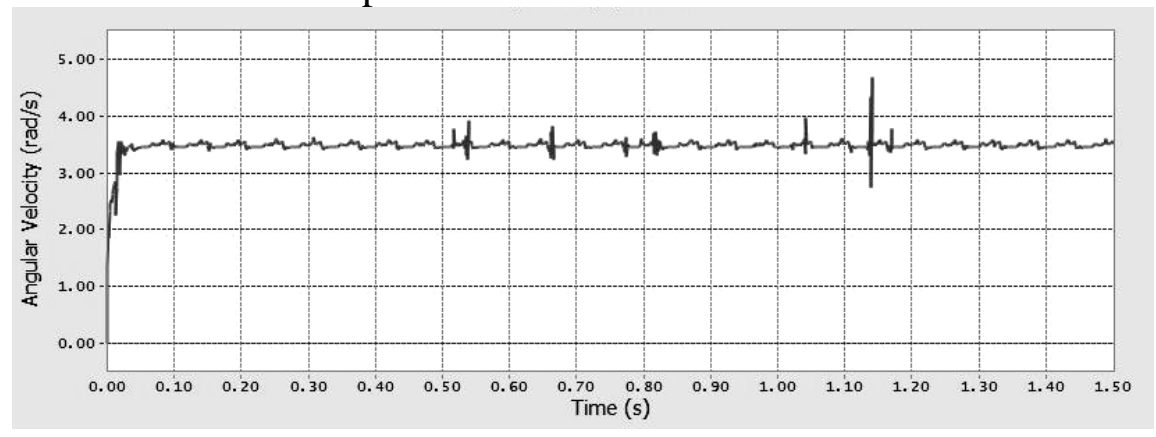

Fig.5 The angular velocity of the planet carrier

Analysis:From the angular velocity of the planet carrier ,we can gain that the curve of the angular velocity of the planet carrier is closed to the theoretical value ,but present a fluctuating change process, at the $0.02 \mathrm{~s}$, the velocity of the planet carrier increase rapidly from 0 to 3.5 , at the $1.14 \mathrm{~s}$,the angular velocity of the planet carrier is increasing at a full speed, in the process of the spinning ,at $0.53 \mathrm{~s}, 0.64 \mathrm{~s}, 1.04 \mathrm{~s}$, etc , the velocity of the planet gear is unstable.

These changes of the velocity will cause the planet carrier deformation,if the time is long ,the deformation of the planet carrier will destroy structure of the planet carrier[4], as well ,it also will destroy transmission accuracy of the planted carrier .These deformation will reduce the service of the carrier,also. We can adopt the special measures according to the curve of the velocity.

\section{Summary}

Results indicate ,the values of the flexible contact force are related to the speed of the mating gear ,tooth,transmission ratio ,etc. We can through the flexible body model ing and simulation to know the circumstance of the gear ,then adopt the effective measures to maintain the planet gear at regular intervals. These study results also provide a reference for the manufacturing process and the late maintenance[5],at the same time, it also save the material and increase the working life of the planet gear of certain type planet gear transmission box.

\section{References}

[1]Yi Liu ,Kai Xu , Jishun Li,et al.The RecurDyn multi-body dynamic simulation And application. Electronic Industry Press,2013,p.269-299.

[2]Zongtang Sun ,A Dissertation for the Degree of M.Eng Researching of the the Rigid-flexible coupled system analytical dynamics modeling. Master of Engineering,Harbin Engineering University,China.p.42-56.

[3]Linying He,Dynamic Load Analysis for Transmission Housing Based on Rigid- Flexible Vehicle Model. Master of Engineering, Jilin University, China.p.59-74. [4]Guoyang Yu,Shixiong Zheng,Yanming Sun.Research On the Method of FlexibleModeling forComplex Engineering.Machine tool and hydraulic .Vol.40(2002). No.2,p.152-153.

[5]Shouqun Sun,Wei Xu,Yuxiang Zhao,et al. Multi-deformable-body dynamics analysis on the ring gear of planetary gear set. Machine design.Vol.26(2009). No.11,p51-54. 